\title{
Scalar and vector decomposition of the nucleon self-energy in the relativistic Brueckner approach
}

\author{
C. Fuchs, T. Waindzoch, Amand Faessler, and D.S. Kosov \\ Institut für Theoretische Physik der Universität Tübingen, \\ D-72076 Tübingen, Germany
}

\begin{abstract}
We investigate the momentum dependence of the nucleon self-energy in nuclear matter. We apply the relativistic Brueckner-Hartree-Fock approach and adopt the Bonn A potential. A strong momentum dependence of the scalar and vector self-energy components can be observed when a commonly used pseudo-vector choice for the covariant representation of the T-matrix is applied. This momentum dependence is dominated by the pion exchange. We discuss the problems of this choice and its relations to on-shell ambiguities of the T-matrix representation. Starting from a complete pseudo-vector representation of the T-matrix, which reproduces correctly the pseudo-vector pion-exchange contributions at the Hartree-Fock level, we observe a much weaker momentum dependence of the self-energy. This fixes the range of the inherent uncertainty in the determination of the scalar and vector self-energy components. Comparing to other work, we find that extracting the self-energy components by a fit to the single particle potential leads to even more ambiguous results.
\end{abstract}

Keywords : nuclear matter, relativistic Brueckner-Hartree-Fock, self-energy

21.30.+y, 21.65.+f, 24.10.Cn

\section{INTRODUCTION}

Concerning the nuclear many-body problem the relativistic Dirac-Brueckner-HartreeFock (DBHF) approach turned out to be remarkably successful in describing the nuclear 
matter saturation mechanism. The major improvement compared to non-relativistic treatments is based on an additional density dependence introduced in the formalism by using a self-consistent spinor basis. To solve the Bethe-Salpeter equation, i.e. its three-dimensional reductions, a variety of approaches relying on different techniques and various bare nucleonnucleon interactions have been developed over the last decade [1] are able to describe reasonably well, although not excellent, nuclear matter properties. In the meantime two main results can be regarded as settled: first, the nuclear single particle potential originates from the cancelation of large repulsive vector and attractive scalar fields, and second, the magnitude of the effective mass is reduced to a value of about $\sim 0.6 M$ at saturation density. Both findings are consistent with effective hadron field theories [11] where, e.g. the effective mass can be determined from the spin-orbit interaction in finite nuclei [12]. Refined treatments which take into account hole-hole excitations [13] or include an additional scaling of meson properties in the nuclear medium [14] do not significantly alter these results.

To test the DBHF approach over a wider range of physical problems also finite nuclei have been studied within effective Hartree-Fock calculations [15] and within a density dependent hadron field theory [16],17]. For a successful application of DBHF results to heavy ion collisions using relativistic transport models, however, it is required to go beyond a local density approximation and to properly account for the non-equilibrium features of the highly anisotropic phase space configurations in such reactions [18]. As soon as nuclei overlap and particles are positioned at close quarters in configuration space, the momentum dependence of the nuclear self-energy starts to play a crucial role in the description of heavy ion collisions. Unfortunately, the determination of the momentum dependence of the nucleon self-energy is a subtle problem in the DBHF approach which has not yet led to settled results. Generally, the techniques applied in the standard relativistic Brueckner approach rely on a weak momentum dependence of the self-energy inside the Fermi sea. This assumption was supported by various calculations in the past [1-3]. In Ref. [4] it was noted that the determination of the self-energy leads to ambiguities arising from the projection of the T-matrix onto positive 
energy states and that the actual momentum dependence strongly depends on the choice of the used nucleon-nucleon interaction 9]. These ambiguities can be avoided when negative energy states are taken into account in the calculation [3,8, 19]. However, the conventional nucleon-nucleon potentials [20] are determined for particle-particle scattering and an extrapolation to anti-particles is in itself ambiguous. To avoid the latter ambiguity we will restrict our discussion merely to the particle sector.

In practice there exist various procedures to determine the self-energy. In the method proposed by Horowitz and Serot [四] one projects onto Lorentz-invariant amplitudes of the T-matrix (or in-medium G-matrix) [1, 2, 4, 7, 9]. This method is, however, not unique since pseudo-scalar (PS) and pseudo-vector (PV) matrix elements are equivalent for on-shell particles in the positive energy sector. Hence, it is impossible to disentangle pseudo-scalar and pseudo-vector contributions in the on-shell T-matrix. On the other hand, the pion exchange contribution is known to react rather sensitively on the particular choice of its representation [21,11], i.e. PS or PV. Thus in the past different choices for the representation of the corresponding amplitude have been used in the relativistic Brueckner scheme [1,2,4]. We find that these choices strongly influence the magnitude and in particular the momentum dependence of the self-energy in the nuclear medium. In the present work we will discuss various possibilities to decompose the T-matrix which are based on the general representation of covariant amplitudes proposed in Refs. [1] and [21]. A strong momentum dependence of the self-energy is found to originate from pseudo-scalar admixtures due to pion-exchange contributions. These pseudo-scalar admixtures are still present when the so called 'pseudo-vector choice' is applied, as it was done in Refs. [2, [, 7,9]. Suppressing the undesirable admixtures by making use of a complete pseudo-vector representation, as proposed in Ref. [21], we find a much weaker momentum dependence of the self-energy components.

In an altogether different approach pursued in Refs. [5, 10], the self-energy components are determined indirectly by a fit to the single particle potential. Here one circumvents the ambiguities of the projection methods. The problem is, however, only shifted to another level of ambiguity, since one has to extract two functions out of one. Thus, the fit method 
leads to highly ambiguous results concerning the momentum dependence of the self-energy components.

The present paper is organized as follows: in section II we review the structure of the nucleon self-energy in nuclear matter. Thereafter we describe in section III the representation of the T-matrix by Lorentz invariant amplitudes and discuss the different on-shell equivalent choices used in the literature. In section IV we present new results obtained for the different choices of the T-matrix representation utilizing the Bonn A potential as the bare nucleonnucleon potential. There we also discuss, as a side remark, the approach pursued in Ref. [5.10]. The paper ends with a summary and the conclusions of our work.

\section{NUCLEON SELF-ENERGY IN NUCLEAR MATTER}

The properties of dressed nucleons in nuclear matter are expressed by the self-energy which enters the in-medium nucleon propagator as the formal solution of the Dyson equation

$$
G(k)=\frac{1}{\not k-M-\Sigma(k)+i \epsilon}
$$

Due to translational and rotational invariance, parity conservation and time reversal invariance the self-energy in isospin saturated nuclear matter has the general form $\Sigma=\Sigma_{s}-\gamma_{\mu} \Sigma^{\mu}$. It depends on the Lorentz invariants $k^{2}, k \cdot j$ and $j^{2}$, with $j_{\mu}$ and $k_{\mu}$ being the baryon current and the nucleon four-momentum, respectively [11]. The invariants can also be expressed in terms of $k_{0},|\mathbf{k}|$ and $k_{\mathrm{F}}$, where $k_{\mathrm{F}}$ denotes the Fermi momentum. Furthermore the vector part of the self energy has contributions proportional to $k^{\mu}$ and to the current $j^{\mu}$. Defining the streaming velocity as $u^{\mu}=j^{\mu} / \sqrt{j^{2}}$, the momentum $k^{\mu}$ can be decomposed into contributions parallel and perpendicular to the streaming velocity, i.e. $k^{\mu}=(k \cdot u) u^{\mu}+\Delta^{\mu \nu} k_{\nu}$ with the projector $\Delta^{\mu \nu}=g^{\mu \nu}-u^{\mu} u^{\nu}$. The vector part of the self-energy can then be written covariantly as 6,22

$$
\Sigma^{\mu}=\Sigma_{\mathrm{o}} u^{\mu}+\Sigma_{\mathrm{v}} \Delta^{\mu \nu} k_{\nu} .
$$

Thus the full self-energy reads 


$$
\begin{aligned}
\Sigma\left(k, k_{\mathrm{F}}\right) & =\Sigma_{\mathrm{S}}\left(k, k_{\mathrm{F}}\right)-\gamma_{\mu}\left[\Sigma_{\mathrm{o}}\left(k, k_{\mathrm{F}}\right) u^{\mu}+\Sigma_{\mathrm{v}}\left(k, k_{\mathrm{F}}\right) \Delta^{\mu \nu} k_{\nu}\right] \\
& =\Sigma_{\mathrm{S}}\left(k, k_{\mathrm{F}}\right)-\gamma_{0} \Sigma_{\mathrm{o}}\left(k, k_{\mathrm{F}}\right)+\left.\gamma \cdot \mathbf{k} \Sigma_{\mathrm{v}}\left(k, k_{\mathrm{F}}\right)\right|_{\mathrm{RF}}
\end{aligned}
$$

where the subscript RF indicates the respective expressions in the nuclear matter rest frame $\left(u^{\mu}=\delta^{\mu 0}\right)$ [1,2]. The $\Sigma_{\mathrm{s}}, \Sigma_{\mathrm{o}}$ and $\Sigma_{\mathrm{v}}$ components are Lorentz scalar functions which actually depend on $k_{0},|\mathbf{k}|$ and $k_{\mathrm{F}}$. They follow from the self-energy matrix by taking the respective traces 22]

$$
\begin{aligned}
& \Sigma_{\mathrm{s}}=\frac{1}{4} \operatorname{tr}[\Sigma] \\
& \Sigma_{\mathrm{o}}=\frac{-1}{4} \operatorname{tr}\left[\gamma_{\mu} u^{\mu} \Sigma\right]=\frac{-1}{4} \operatorname{tr}\left[\gamma_{0} \Sigma\right]_{\mathrm{RF}} \\
& \Sigma_{\mathrm{v}}=\frac{-1}{4 \Delta^{\mu \nu} k_{\mu} k_{\nu}} \operatorname{tr}\left[\Delta^{\mu \nu} \gamma_{\mu} k_{\nu} \Sigma\right]=\frac{-1}{4|\mathbf{k}|^{2}} \operatorname{tr}[\gamma \cdot \mathbf{k} \Sigma]_{\mathrm{RF}} .
\end{aligned}
$$

The Dirac equation for the in-medium spinor basis can be deduced from the Green function (1). Written in terms of effective masses and momenta

$$
M^{*}=M+\operatorname{Re} \Sigma_{\mathrm{s}} \quad, \quad k_{\mu}^{*}=k_{\mu}+\operatorname{Re} \Sigma_{\mu}
$$

the Dirac equation has the form

$$
\left[k^{*}-M^{*}-i \operatorname{Im} \Sigma\right] u(k)=0 .
$$

In the following we will work in the quasi-particle approximation and therefore we neglect the imaginary part of the self-energy from now on. Thus the effective nucleon four-momentum will be on mass shell even above the Fermi surface, fulfilling the relation $k_{\mu}^{*} k^{* \mu}=M^{* 2}$. Since we only deal with the real part of the self-energy in the quasi-particle approximation we omit this in the notation. In the nuclear matter rest frame the four-momentum follows from Eq. (8)

$$
\mathbf{k}^{*}=\mathbf{k}\left(1+\Sigma_{\mathrm{v}}\right) \quad, \quad k_{0}^{*}=E^{*}=\sqrt{\mathbf{k}^{2}\left(1+\Sigma_{\mathrm{v}}\right)^{2}+M^{* 2}}
$$

which allows one to eliminate the $\Sigma_{\mathrm{v}}$-term in the Dirac equation,

$$
\left[(\alpha \cdot \mathbf{k})-\gamma^{0} \tilde{M}^{*}\right] u(k)=\tilde{E}^{*} u(k)
$$


by a rescaling of effective mass and energy

$$
\tilde{M}^{*}=\frac{M^{*}}{1+\Sigma_{\mathrm{v}}} \quad, \quad \tilde{E}^{*}=\frac{E^{*}}{1+\Sigma_{\mathrm{v}}}=\sqrt{\mathbf{k}^{2}+\tilde{M}^{* 2}} .
$$

From the Dirac equation in the form (11) one derives the in-medium relativistic Hamiltonian for nucleons and thereby the operator for the single-particle potential within the DBHF approximation, i.e. $\hat{U}=\gamma^{0} \Sigma$. The expectation value of $\hat{U}$, i.e. sandwiching $\hat{U}$ between the effective spinor basis, yields the single particle potential

$$
U(k)=\frac{<u(k)\left|\gamma^{0} \Sigma\right| u(k)>}{<u(k) \mid u(k)>}=\frac{M^{*}}{E^{*}(\mathbf{k})}<\bar{u}(k)|\Sigma| u(k)>
$$

which can be evaluated as

$$
\begin{aligned}
U\left(k, k_{\mathrm{F}}\right) & =\frac{M^{*}}{E^{*}} \Sigma_{\mathrm{s}}-\frac{k_{\mu}^{*} \Sigma^{\mu}}{E^{*}} \\
& =\frac{M^{*} \Sigma_{\mathrm{s}}}{\sqrt{\mathbf{k}^{2}\left(1+\Sigma_{\mathrm{v}}\right)^{2}+M^{* 2}}}-\Sigma_{\mathrm{o}}+\frac{\left(1+\Sigma_{\mathrm{v}}\right) \Sigma_{\mathrm{v}} \mathbf{k}^{2}}{\sqrt{\mathbf{k}^{2}\left(1+\Sigma_{\mathrm{v}}\right)^{2}+M^{* 2}}} .
\end{aligned}
$$

In many applications [5, 10] the single particle potential is only given in terms of a scalar and zero-vector component. This can be achieved in nuclear matter by introducing reduced fields $\tilde{\Sigma}_{\mathrm{s}}$ and $\tilde{\Sigma}_{\mathrm{o}}$ as

$$
\tilde{\Sigma}_{\mathrm{s}}=\tilde{M}^{*}-M=\frac{\Sigma_{\mathrm{s}}-\Sigma_{\mathrm{v}} M}{1+\Sigma_{\mathrm{v}}} \quad, \quad \tilde{\Sigma}_{\mathrm{o}}=\tilde{E}^{*}-E=\Sigma_{\mathrm{o}}-\tilde{E}^{*}(\mathbf{k}) \Sigma_{\mathrm{v}} .
$$

Then the single particle potential has the form

$$
U\left(k, k_{\mathrm{F}}\right)=\frac{\tilde{M}^{*}}{\tilde{E}^{*}} \tilde{\Sigma}_{\mathrm{s}}-\tilde{\Sigma}_{\mathrm{o}}
$$

Frequently the reduced fields, Eq. (16), are used rather than the projected components since they represent the self-energy in a mean field or Hartree form. Thus they can easily be related to effective hadron mean field theory [16,17]. Such a representation is meaningful since the $\Sigma_{\mathrm{v}}$-contribution is a moderate correction.

In contrast to the single particle potential which can easily be derived form the T-matrix [5] the extraction of the self-energy components is a subtle problem. In the latter case one has to represent the T-matrix within the Clifford algebra in the Dirac space which is 
not free from severe ambiguities. Before we discuss this point in detail we briefly recall some basic features of the relativistic Brueckner scheme. For more details see e.g. Refs. [1:2].9]. The iteration of the Thompson equation requires to determine the self-consistent spinor basis, Eq. (9). In practice the problem is treated in terms of the reduced effective

mass $\tilde{M}^{*}$ to avoid an explicit dependence on the space-like $\Sigma_{\mathrm{v}}$ components (11). Actually, the zero-vector component $\Sigma_{\mathrm{o}}$ does not enter the self-consistency problem. In the standard treatment [1,2,5,6,9] the effective mass is assumed to be entirely density dependent, i.e. a constant effective mass $\tilde{M}^{*}=\tilde{M}^{*}\left(|\mathbf{k}|=k_{\mathrm{F}}, k_{\mathrm{F}}\right)$ generally taken at the Fermi-momentum is used as the iteration parameter. The effective mass at its value at the Fermi momentum is reinserted into the Thompson equation and this procedure is repeated until convergence is reached. Such a treatment is self-consistent concerning the density dependence of the effective interaction screened by the medium and appears to be justified if the self-energy is weakly momentum dependent inside the Fermi-sea. If the self-energy is, however, strongly momentum dependent, as it was observed in [4,9], one principally has to go beyond the standard approach. Then one has to include the momentum dependence of the effective mass in the Thompson propagator as well as on the level of the interaction. For the present investigations we will not include such an explicit momentum dependence in the Brueckner scheme since first of all, this is technically a rather involved problem. Secondly, we will verify in the next section that the momentum dependence of the self-energy is mainly governed by the treatment of the pion-exchange in the T-matrix representation. Thus a more careful treatment of the pion-exchange leads to a much weaker momentum dependents of the selfenergy as it is desirable for the present self-consistency scheme.

\section{DECOMPOSITION BY LORENTZ INVARIANT AMPLITUDES}

The self-energy for the nucleon $k$ follows from the T-matrix by integrating over the occupied states $q$ in the Fermi sea

$$
\Sigma(k)=-i \int \frac{d^{4} q}{(2 \pi)^{4}} \operatorname{tr}\left[G_{\mathrm{D}}(q) \hat{T}(q k ; q k-k q)\right] .
$$


Here $\hat{T}$ denotes the T-matrix (or G-matrix) operator depending on four Dirac indices of the ingoing and outgoing nucleons. Due to antisymmetrization $\hat{T}$ contains a direct ( Hartree) and an exchange (Fock) contribution. The Dirac propagator

$$
G_{\mathrm{D}}(q)=\left[q^{*}+M^{*}\right] 2 \pi i \delta\left(q^{* 2}-M^{* 2}\right) \Theta\left(q^{* 0}\right) \Theta\left(k_{\mathrm{F}}-|\mathbf{q}|\right)
$$

projects onto positive energy states in the Fermi sea. In order to project out the self-energy components, Eqs. (5-7), the T-matrix has to be decomposed into Lorentz invariants. Since we need to consider only on-shell scattering of particles in eq. (18), five invariant amplitudes with five covariants are sufficient to represent the on-shell T-matrix [21]. In this case, the scalar, vector, tensor, axial-vector and pseudo-scalar terms

$S=1 \otimes 1 \quad, \quad V=\gamma^{\mu} \otimes \gamma_{\mu} \quad, \quad T=\sigma^{\mu \nu} \otimes \sigma_{\mu \nu} \quad, \quad A=\gamma_{5} \gamma^{\mu} \otimes \gamma_{5} \gamma_{\mu} \quad, \quad P=\gamma_{5} \otimes \gamma_{5}$

form a linearly independent, however, not unique set of covariants. Using this special set, the on-shell T-matrix can be represented entirely by 'direct' amplitudes

$$
\hat{T}(\theta)=F_{1}(\theta) S+F_{2}(\theta) V+F_{3}(\theta) T+F_{4}(\theta) A+F_{5}(\theta) P
$$

The covariant amplitudes $F_{i}$ are determined from anti-symmetrized plane wave helicity matrix amplitudes [20] which obey the selection rule $(-)^{L+S+I}=-1$. We solve the relativistic Thompson equation for the T-matrix [9] consistently in the two-particle center-of-mass frame. Since we need only diagonal matrix elements for the self-energy (18) the $F_{i}$ amplitudes are required at the scattering angle $\theta=0$. They are already anti-symmetrized and contain implicitly direct and exchange contributions. Therefore the simple representation (21) is sufficient to calculate the self-energy. If we insert the T-matrix given by Eq. (21) into Eq. (18) and take the trace over the Dirac propagator only the scalar and vector amplitudes $F_{1}$ and $F_{2}$ survive and contribute to the self-energy,

$$
\Sigma(k)=-i \int \frac{d^{3} q}{(2 \pi)^{3}} \frac{\Theta\left(k_{\mathrm{F}}-|\mathbf{q}|\right)}{E^{*}(\mathbf{q})}\left[m^{*} F_{1}(0)+q^{*} F_{2}(0)\right] .
$$

The representation of $\hat{T}$ is, however, not uniquely defined in the on-shell case and therefore various alternative possibilities exist to construct the set of five independent covariants in 
the subspace of positive energies. Although the different representations discussed below are all equivalent if one works with the pseudo-scalar covariant $P$, their difference becomes crucial as soon as one switches from the pseudo-scalar to the pseudo-vector representation. The PV covariant in the medium is defined as

$$
P V=\frac{\not k_{2}^{*}-\not k_{1}^{*}}{2 M^{*}} \gamma_{5} \otimes \frac{\not_{2}^{*}-\not q_{1}^{*}}{2 M^{*}} \gamma_{5}
$$

with $k_{1}^{*}, q_{1}^{*}$ the initial and $k_{2}^{*}, q_{2}^{*}$ the final momenta of the scattering particles. The ambiguity of the decomposition procedure arises from the fact that the PS and PV matrix elements are identical (using the Dirac eq.) for on-shell scattering of positive energy states, i.e.

$$
\bar{u}\left(k_{2}\right)\left(\frac{k_{2}^{*}-\not k_{1}^{*}}{2 M^{*}}\right) \gamma_{5} u\left(k_{1}\right)=\bar{u}\left(k_{2}\right) \gamma_{5} u\left(k_{1}\right)
$$

Thus the corresponding amplitudes are identical as well and it is impossible to uniquely disentangle the PS and PV contributions in the T-matrix.

However, it is known from meson theory of the nuclear interaction that a pseudo-vector representation of the $\pi N$ coupling is preferable [20]. The influence of the pion in the relativistic theory has been discussed in detail, e.g. in Refs. [11,21]. There it was shown that the one-pion exchange contribution to the nuclear optical potential tends to increase drastically at low momenta if the $\pi N$ vertex is treated as PS. One reason for this behavior is a strong PS coupling to negative energy states which is not apparent in non-relativistic approaches. The PV vertex suppresses the coupling to antiparticles since the overlap matrix elements vanish for on-shell scattering

$$
\bar{v}\left(k_{2}\right)\left(\frac{\not k_{2}^{*}-\not k_{1}^{*}}{2 M^{*}}\right) \gamma_{5} u\left(k_{1}\right)=0
$$

Therefore a PV vertex is more consistent with the approximation scheme of the conventional Brueckner model where the coupling to negative energy states is not taken into account. The PV vertex also strongly suppresses the pion contribution in particular at low energies which is more in accordance with the empirical knowledge from proton-nucleus scattering [11,21]. Consequently, the $\pi N$ vertex of the bare interaction is usually treated as PV [20]. 
Due to these facts the usage of a PV covariant in the decomposition of the T-matrix is considered as preferable [2,9]. However, simply replacing $P$ by $P V$ in Eq. (21) leads to identical results; firstly, because the corresponding amplitudes are equal and secondly, because only the scalar and vector amplitudes $F_{1}$ and $F_{2}$ contribute to the self-energy (22). Motivated by this fact alternative representation of the T-matrix have been used [2, 4, 9] which lead to an - in principle superfluous - explicit splitting into 'direct' and 'exchange' contributions and give different results when the $P S \longmapsto P V$ replacement is performed.

\section{A. Symmetrized representations}

Based on Ref. [23] Tjon and Wallace discussed a representation which accounts for the structure of the exchange contributions in $\hat{T}$ in a more transparent way [21]. To express exchange contributions also the Dirac indices of the covariants are interchanged. The transfor-

mation $\tilde{S}$ interchanges the Dirac indices of particles 1 and 2, i.e. $\tilde{S} u(1)_{\sigma} u(2)_{\tau}=u(1)_{\tau} u(2)_{\sigma}$. Thus one obtains the interchanged covariants as $\tilde{S}=\tilde{S} S, \tilde{V}=\tilde{S} V, \tilde{T}=\tilde{S} T, \tilde{A}=\tilde{S} A$ and $\tilde{P}=\tilde{S} P$. The interchanged covariants are related to the original covariants (21) by a Fierz transformation $\mathcal{F}[21]$

$$
\left(\begin{array}{c}
\tilde{S} \\
\tilde{V} \\
\tilde{T} \\
\tilde{A} \\
\tilde{P}
\end{array}\right)=\frac{1}{4}\left(\begin{array}{ccccc}
1 & 1 & \frac{1}{2} & -1 & 1 \\
4 & -2 & 0 & -2 & -4 \\
12 & 0 & -2 & 0 & 12 \\
-4 & -2 & 0 & -2 & 4 \\
1 & -1 & \frac{1}{2} & 1 & 1
\end{array}\right)\left(\begin{array}{c}
S \\
V \\
T \\
A \\
P
\end{array}\right)
$$

For a definite scattering angle $\theta$ the T-matrix is now represented by five symmetrized covariants [21]

$$
\hat{T}(\theta)=f_{1}(\theta)(S-\tilde{S})+f_{2}(\theta) \frac{1}{2}(T+\tilde{T})-f_{3}(\theta)(A-\tilde{A})+f_{4}(\theta)(V+\tilde{V})+f_{5}(\theta)(P-\tilde{P})
$$

These symmetrized covariants are constructed so that the amplitudes $f_{i}$ account for the Pauli principle in a transparent way. Interchanging the outgoing particles, anti-symmetrization 
requires the following symmetry

$$
f_{i}^{\mathrm{I}}(\pi-\theta)=(-)^{\mathrm{I}+\mathrm{i}} f_{i}^{\mathrm{I}}(\theta)
$$

for definite isospin $I=0,1$.

The relation between the five new amplitudes $f_{i}$ and the former amplitudes $F_{i}$ is given by the transformation 21

$$
\left(\begin{array}{l}
f_{1} \\
f_{2} \\
f_{3} \\
f_{4} \\
f_{5}
\end{array}\right)=\frac{1}{4}\left(\begin{array}{ccccc}
2 & -4 & -12 & 0 & 0 \\
1 & 0 & 4 & 0 & 1 \\
0 & -2 & 0 & -2 & 0 \\
1 & 2 & 0 & -2 & -1 \\
0 & 4 & -12 & 0 & 2
\end{array}\right)\left(\begin{array}{l}
F_{1} \\
F_{2} \\
F_{3} \\
F_{4} \\
F_{5}
\end{array}\right) .
$$

With relation (28) one can express $\mathrm{T}$ as the combination of two terms which resemble a direct and an exchange contribution, i.e. $\hat{T}=\hat{T}^{\mathrm{D}}-\hat{T}^{\mathrm{x}}$, by

$$
\begin{aligned}
& \hat{T}^{\mathrm{D}}(\theta):=f_{1}(\theta) S+f_{2}(\theta) \frac{1}{2} T-f_{3}(\theta) A+f_{4}(\theta) V+f_{5}(\theta) P \\
& \hat{T}^{\mathrm{X}}(\theta):=(-)^{\mathrm{I}+1}\left[f_{1}(\pi-\theta) \tilde{S}+f_{2}(\pi-\theta) \frac{1}{2} \tilde{T}-f_{3}(\pi-\theta) \tilde{A}+f_{4}(\pi-\theta) \tilde{V}+f_{5}(\pi-\theta) \tilde{P}\right] .
\end{aligned}
$$

However, it can not be claimed that $\hat{T}^{\mathrm{D}}$ and $\hat{T}^{\mathrm{x}}$ are the real direct and exchange matrix elements but only their combination yields the fully anti-symmetrized matrix elements.

The representation proposed by Horowitz and Serot [1] and also used in other works [2.9] is of a similar structure as the one above with the difference that it is based on direct amplitudes

$$
\begin{aligned}
& \hat{T}^{\mathrm{D}}(\theta):=\frac{1}{2}\left[F_{1}(\theta) S+F_{2}(\theta) V+F_{3}(\theta) T+F_{4}(\theta) A+F_{5}(\theta) P\right] \\
& \hat{T}^{\mathrm{x}}(\theta):=(-)^{\mathrm{I}+1} \frac{1}{2}\left[F_{1}(\pi-\theta) \tilde{S}+F_{2}(\pi-\theta) \tilde{V}+F_{3}(\pi-\theta) \tilde{T}+F_{4}(\pi-\theta) \tilde{A}+F_{5}(\pi-\theta) \tilde{P}\right] .
\end{aligned}
$$

To be more precise, in this approach both set of amplitudes $F_{i}(\pi)$ and $F_{i}(\pi-\theta)$ are obtained from the helicity matrix elements applying representation (21) at angle $\theta$ and $\pi-\theta$, respectively. Doing this, the amplitudes $F_{i}$ are essentially different from the $f_{i}$ amplitudes 
of Eq. (27). Instead of relation (28) anti-symmetrization requires now that the exchange amplitudes are connected to the direct amplitudes by the Fierz transformation (26)

$$
F_{i}^{\mathrm{I}}(\pi-\theta)=(-)^{\mathrm{I}} \mathcal{F}_{j i} F_{j}^{\mathrm{I}}(\theta)
$$

The difference between the representations (21) and (31) lies in the fact that Eq. (31) leads to an explicit splitting into direct and exchange contributions which, however, can be regarded as - at least partially - artificial. Since the amplitudes $F_{i}(\theta)$ and $F_{i}(\pi-\theta)$ in (31) are determined from already anti-symmetrized helicity matrix elements one has in that case the identity

$$
\hat{T}^{\mathrm{X}}=-\hat{T}^{\mathrm{D}}
$$

Hence the normalization factor $1 / 2$ which determines the splitting into 'direct' and 'exchange' parts in (31) is judicious and could also be fixed differently by any normalized linear combination. In this context we want to mention that concerning the original work of Horowitz and Serot [1] this statement would not hold because they used non-antisymmetrized (unphysical) helicity states in their formalism and therefore they had to anti-symmetrize explicitly the T-matrix elements by splitting the representation into direct and exchange contributions as done in Eq. (31). As a consequence, their amplitudes $F_{i}$ at angle $\pi$ and $\pi-\theta$ did not fulfill the anti-symmetry relation (32) and thus only the representation (31) was physically meaningful.

Working with physical helicity states, however, one retains relation (32) and therefore all types of decompositions, Eqs. (21-31), are equivalent as long as one restricts to a pseudoscalar representation. But if one replaces the PS by the PV covariant the equivalence of the expressions (21), (27) and (31) is destroyed. Our 'optimal choice' of the representation for using the PV covariant will be discussed in the next section. 


\section{B. Pseudo-vector representation}

The nucleon-nucleus potential is very sensitive to the treatment of the pseudo-scalar or pseudo-vector treatment of the pion-nucleon interaction. As already mentioned, the pion should be preferentially treated with a pseudo-vector coupling in the bare NN interaction. The corresponding coupling strength is fixed in such a way that it reproduces the on-shell PS coupling strength in the vacuum [20]. This already leads to a suppression of the vertex by a factor $\left(\frac{M^{*}}{M}\right)^{2}$ inside the nuclear medium [11]. However, the major suppression of the pion exchange contribution to the nucleon self-energy originates from the different cofactors which arise if one inserts the Dirac propagator (19) and the T-matrix from (21) or (31) into the Eq. (18) for the self-energy [2.9], i.e.

$$
\begin{aligned}
\operatorname{tr}\left[\left(\phi^{*}+M^{*}\right) \tilde{P}\right] & =-\left(\phi^{*}+M^{*}\right) \\
\operatorname{tr}\left[\left(\phi^{*}+M^{*}\right) \widetilde{P V}\right] & =\left(\not k^{*}+M^{*}\right)\left(\frac{k_{\mu}^{*} q^{* \mu}}{2 M^{* 2}}-\frac{1}{2}\right) .
\end{aligned}
$$

The influence of the pion, in particular the sensitivity on the PS or PV representation, is

most clearly demonstrated at the Hartree-Fock level. This means that $\hat{T}$ is replaced by the bare NN interaction $\hat{V}$ and no further medium effects are taken into account, i.e. bare nucleon masses are used and the Pauli operator in the Thompson equation is neglected. Furthermore, the Hartree-Fock expressions are known analytically [24] which provides also a straightforward check of the involved projection techniques. It is a well known fact [11] that the PS treatment yields extremely large pion contributions to the self-energy whereas the PV representation suppresses these terms almost completely. This effect is demonstrated in Fig. 1 where the Hartree-Fock contributions of the pion only to the nuclear self-energy are shown. The calculations are performed for a nuclear matter density of $\rho=0.166 \mathrm{fm}^{-3}$ with a PS and PV (denoted as 'full PV' in Fig. 1) $\pi$ NN coupling of $g_{\pi}^{2} / 4 \pi=14.9$ and the pion form factor taken from the Bonn A interaction [20]. The bare nucleon mass is used in this example. It is seen that the PS description of the pion exchange yields extremely large self-energy components at low momenta which are rapidly decreasing with increasing 
momentum. The $\Sigma_{\mathrm{s}}$ and $\Sigma_{\mathrm{o}}$ contributions are almost identical which leads to a strong cancelation effect in the single particle potential. The PV description (denoted as 'full PV' in Fig. 1) suppresses the pion by nearly two orders of magnitude and even on this new scale the momentum dependence is much less pronounced. Remarkably, scalar and vector contributions have now opposite signs. Thus they add up in the potential (17) and the remaining momentum dependence is not the remnant of a huge cancelation effect as in the PS case.

For comparison we also show the results which are obtained in the projection scheme when the so-called 'PV choice' is adopted. In the 'PV choice' commonly used [2,9] we simply replace the pseudo-scalar covariants by the pseudo-vector covariants $P, \tilde{P} \longmapsto P V, \widetilde{P V}$ in the decomposition of the T-matrix (31). Due to the on-shell equivalence of the respective matrix elements, Eq. (24) the amplitudes remain thereby unchanged. Now the explicit choice of the T-matrix representation starts to play a decisive role since the PS and PV exchange terms contribute differently to the self-energy, Eqs. (34,35). Thus the strength of the PS $\longmapsto \mathrm{PV}$ replacement, determined by the respective amplitudes $f_{5}(\theta)$ and $F_{5}(\pi-\theta)$, becomes important. In most previous calculations [2,9] the representation (31) was used. The effect of the replacement can be seen in Fig. 1 where the Hartree-Fock contribution of the pion exchange to the nucleon self-energy using the PV choice is shown. First of all, it should be noted that the result using the PV choice for (31) is identical to the result which one obtains if one uses the replacement in the symmetrized representation, Eq. (27), since both amplitudes $f_{5}(\theta)$ and $F_{5}(\pi-\theta)$ agree in the Hartree-Fock approach with only pion exchange. It is obviously transparent from Fig. 1 that within the traditional PV choice the pion is not treated correctly as a pseudo-vector particle. The PV choice representation is rather a mixture of the PS and a complete PV representation. Although the pion contribution to the nucleon self-energy is suppressed by about a factor of two compared to the original PS case, the structure of the self-energies, in particular the pronounced momentum dependence is still very similar. The reason for this behavior of the self-energy is easily understandable. Even after replacing $P, \tilde{P}$ by $P V, \widetilde{P V}$, both representations, Eqs. (27) and (31), still contain 
pseudo-scalar admixtures because the Fierz transformation (26) couples all covariants. This leads to identities for the symmetrized vector and tensor covariants 21]

$$
\begin{aligned}
\frac{1}{2}(T+\tilde{T}) & =S+\tilde{S}+P+\tilde{P} \\
V+\tilde{V} & =S+\tilde{S}-P-\tilde{P} .
\end{aligned}
$$

In order to completely remove the PS part from the interaction one first should use the identities above which leads to the following decomposition [21]

$$
\begin{aligned}
\hat{T}(\theta)= & \left(f_{1}+f_{2}+f_{4}\right) S-\left(f_{1}-f_{2}-f_{4}\right) \tilde{S}-f_{3}(A-\tilde{A}) \\
& +\left(f_{5}+f_{2}-f_{4}\right) P-\left(f_{5}-f_{2}+f_{4}\right) \tilde{P}
\end{aligned}
$$

If the replacement $P, \tilde{P} \longmapsto P V, \widetilde{P V}$ is now performed in Eq. (38) instead of Eq. (27) or Eq. (31) this yields a complete PV representation of the interaction which we will call 'full PV' representation in Fig. 1 and in the following. Such a decomposition can successfully describe the PV pion exchange on the Hartree-Fock level, i.e. the results calculated with the analytically known Hartree-Fock matrix elements 24 for the PV pion-exchange are reproduced. In the present formalism this can not be achieved by the other decompositions. On the other hand, however, e.g. the $\omega$ exchange is no longer treated accurately in the full PV representation since PS admixtures arising from the Fock part of the $\omega$ exchange, Eq. (37), are treated as PV. This bias will, however, turn out to be small. Anyway, all existing decomposition are unable to handle the PV pion exchange simultaneously with the remaining set of mesons on the Hartree-Fock level. As we will see later on, the one-pion exchange dominates the momentum dependence of the self-energy. Hence, it is reasonable to require that the PV pion-exchange is treated exactly on the Hartree-Fock level. This constraint is fulfilled adopting the full PV treatment.

\section{RESULTS}

In the present section we study the impact of the various representations of the T-matrix (21), (27), (31) and (38) on the nucleon self-energy and on related quantities. 


\section{A. Momentum dependence of the self-energy}

On the level of the self-energy the effect of the different choices can be summarized as

$$
\Sigma(k)=\Sigma^{\mathrm{PS}}(k)-\delta \Sigma(k)=\Sigma^{\mathrm{PS}}(k)+i \int \frac{d^{4} q}{(2 \pi)^{4}} f_{R}(k q ; q k) \operatorname{tr}\left[G_{\mathrm{D}}(q)(\tilde{P}-\widetilde{P V})\right]
$$

with $\Sigma^{\mathrm{PS}}$ being the self-energy given in the pure PS representation. The different approaches for the self-energy $\Sigma$ using for the T-matrix Eqs. (21), (27), (31) or (38) are only varying in the choice of $f_{R}$ explained below. The strength of the suppression of the pseudo-scalar contributions is moderated by the $f_{R}$ amplitude which also determines the deviation $\delta \Sigma$ of the self-energy from the PS case. In principle this deviation shift is only apparent in the decomposition of $\Sigma$ in the scalar and vector components, Eq. (3), but vanishes when the complete matrix elements are built, i.e.

$$
<\bar{u}(k)|\delta \Sigma(k)| u(k)>=0
$$

For the same effective mass $M^{*}\left(k_{\mathrm{F}}\right)$ all approaches give the same total single particle potential (13) although they yield quite different scalar and vector self-energy components. However, the different approaches also yield different values for the effective mass $M^{*}\left(k_{\mathrm{F}}\right)$ which leads to a different in-medium spinor basis $\mid u>$ used in the self-consistent iteration procedure and the equivalence for the single particle potential gets distorted. Consequently, the different approaches lead to visuable changes also for those quantities which are built from total matrix elements, i.e. the single particle potential and the equation-of-state. If the value of $M^{*}$ is, however, kept fixed the equivalence on the level of matrix elements is exact and also numerically fulfilled with high accuracy. The cases discussed in the previous section modify only $f_{R}$ in Eq. (39) and can now be summarized as

$$
f_{R}=\left\{\begin{array}{ccc}
0 & , & \mathrm{PS} \\
(-)^{\mathrm{I}+1} \frac{1}{2} F_{5}(\pi-\theta) & , & \text { 'PV choice' } \\
f_{5}(\theta)-f_{2}(\theta)+f_{4}(\theta) & , & \text { 'full } \mathrm{PV}^{\prime}
\end{array}\right.
$$

Since earlier works on relativistic Brueckner theory which sticked to the projection method [2.44,9] applied the scheme proposed by Horowitz and Serot (Eq. (31) with $P$ and $\tilde{P}$ replaced 
by $P V$ and $\widetilde{P V}$, respectively, our 'PV choice') we will discuss this method first. Within this scheme the structure of the self-energy, i.e. its density and momentum dependence has been investigated in detail in Ref. [9] with the Bonn potentials [20] as the bare NNinteraction. As the most prominent result we observed a strong momentum dependence of the scalar and time-like vector self-energy components around the Fermi momentum. These findings are in qualitative agreement with a previous work by Nuppenau et al. 迁. Fig. 2 shows the momentum dependence of the three self-energy components $\Sigma_{\mathrm{s}}, \Sigma_{\mathrm{o}}, k_{\mathrm{F}} \Sigma_{\mathrm{v}}$ at nuclear matter density $\rho=0.166 \mathrm{fm}^{-3}$ obtained with the Bonn A interaction. The space-like $\Sigma_{\mathrm{v}}$ component is found to be relatively large inside the Fermi-sea and decreases with increasing momentum. Furthermore we compare to the corresponding reduced fields $\tilde{\Sigma}_{\mathrm{S}}, \tilde{\Sigma}_{\mathrm{O}}$ where the $\Sigma_{\mathrm{v}}$-term is effectively included, Eq. (16). It is seen that the reduced fields are significantly smaller in magnitude at low momenta and generally show a less pronounced momentum dependence. The inclusion of the $\Sigma_{\mathrm{v}}$-term counterbalances the strong momentum dependence to some extent. The negative $\Sigma_{\mathrm{v}}$-contribution effectively weakens the momentum dependence whereas in the opposite case 66,19] the momentum dependence will be enhanced. In the limit of a vanishing $\Sigma_{\mathrm{v}}$-contribution reduced and projected components are equal.

Fig. 3 demonstrates the influence of the various mesons (using Bonn A). Taking only $\sigma$ and $\omega$ exchange into account the result is quite similar to that obtained in Ref. [1], i.e. the momentum dependence is flat inside the Fermi sea. Including the pion we are already very close to the full result. The strong momentum dependence of the present calculation originates to a large extent from the pion-exchange. Hence, the calculation still reflects the Hartree-Fock results (Fig.11) and the strong momentum dependence originates mainly from pseudo-scalar contributions of the pion.

In Fig. the corresponding self-energies obtained for the various decompositions are compared. Adopting the full PV representation the space-like $\Sigma_{\mathrm{v}}$ contribution turns out to be much smaller than in the PS or the standard PV choice (see also Fig. 2). Therefore we show 
the reduced self-energies $\tilde{\Sigma}_{\mathrm{s}}$ and $\tilde{\Sigma}_{\mathrm{o}}$ in which $\Sigma_{\mathrm{v}}$ is included for a better comparison. Both, the PS and the PV choice show a similar strong momentum dependence which again reflects the pseudo-scalar nature of the pion exchange. Consistent with the previous considerations this momentum dependence vanishes to a large extent when the pion contribution is suppressed by the full PV representation of the T-matrix. At high energies the different choices yield similar results since the influence of the pion decreases. The pure PS and the full PV representation can be regarded as the limiting cases which give the range of uncertainty in the determination of the self-energy. The full PV representation has thereby the big advantage that due to the weak momentum dependence the standard treatment of the Thompson equation, i.e. to approximate the effective mass by its constant value at the Fermi surface, can be safely applied as done in our and almost all other works on this subject. Furthermore this method ensures by construction that it treats the pion correctly at the Hartree-Fock level. Thus the full PV representation comes closest of all discussed representations to what we would call the optimal representation of the T-matrix. It is also worthwhile to mention that the results obtained within this representation agree well with recent calculations which include negative energy states and thus avoid the projection procedure [19].

\section{B. Single particle potential and the fit method}

As discussed above, the single particle potential, Eq. (13), is in principle independent on the representation of the T-matrix which is again due to the on-shell equivalence of the corresponding matrix elements, Eq. (24). However, significantly different values of $M^{*}$ obtained in the different approaches lead to different results. Although this effect is reduced using the rescaled effective mass $\tilde{M}^{*}$, the equation-of-state reacts sensitively on this $M^{*}$ dependence, as can be seen from table 1. A suppression of the PS contributions causes a larger effective mass and thus suppresses relativistic effects which originate from the mixing of small and large components of the spinors. A pure PS treatment leads to more binding and shifts the saturation point to higher densities. The corresponding equation-of-state is rather 
stiff. By the empirical knowledge of the nuclear saturation properties the PS representation can be ruled out since the saturation density is much too high and the effective mass is much too small. The remaining two methods are in rough agreement with the empirical constraints, however, the densities are also slightly too high compared to the experimental Fermi momentum of about $k_{\mathrm{F}}=1.37 \mathrm{fm}^{-1}$. Here the larger value of the effective mass is a favor of the 'full PV' representation since it is in better agreement with the constraints derived from the spin-orbit splitting in finite nuclei [12]. Compared to the 'PV choice', the 'full PV' representation leads to more binding and makes the equation-of-state softer, in agreement with the incompressibility derived from the isoscalar giant monopole resonance of about $\mathrm{K} \approx 230 \mathrm{MeV}$. One might assume that this is due to the fact that the 'full PV' representation suppresses part of the repulsive $\omega$-exchange. This is however, not the case since the inaccuracy which arises in the $P S \longmapsto P V$ replacement procedure concerning the vector and tensor exchange amplitudes, Eqs. (37), has only a minor influence on the final results. We checked this point by treating the Hartree-Fock contribution of the one-pionexchange separately in the PV representation while for the remaining part of the T-matrix the PS representation was retained. In terms of the self-energy this means to set $f_{R}=f_{\pi}^{X}$ in (39) with $f_{\pi}^{X}$ the Hartree-Fock amplitude of the one-pion exchange.

In view of the problems which arise in the determination of the self-energy we now briefly discuss a frequently used and much simpler approach first applied by Brockmann and Machleidt [5]. In this approach one tries to extract the self-energy components directly from the single particle potential, thus one avoids to take the explicit traces (5 7). Therefore there is no need to decompose the T-matrix into its Lorentz invariants. Actually, Brockmann and Machleidt determined only density dependent but not momentum dependent mean values for $\tilde{\Sigma}_{\mathrm{s}}, \tilde{\Sigma}_{\mathrm{o}}$ by a fit to $U$ given by Eq. (17). This fit method works reasonably well as long as one restricts oneself to density dependent observables on the one-body level. The corresponding (reduced) effective masses are relatively close to our results at $k_{\mathrm{F}}$ obtained within the 'PV choice' [9]. Thus it is understandable that the resulting nuclear matter saturation properties are similar in the two approaches of Refs. [5] and [9], see Tab.1. However, any information 
on the magnitude of the space-like $\Sigma_{\mathrm{v}}$-contribution and the explicit momentum dependence of the self-energy is completely lost in this approach. To overcome this drawback the Stony Brook group [10] extracted momentum dependent fields from the single particle potential. They assumed a functional dependence of the (reduced) self-energies of the form

$$
\tilde{\Sigma}_{\mathrm{s}, \mathrm{o}}(k)=\frac{\alpha_{\mathrm{s}, \mathrm{o}}}{1+\beta_{\mathrm{s}, \mathrm{o}}\left(k / k_{\mathrm{F}}\right)^{\gamma_{\mathrm{s}, \mathrm{o}}}}
$$

at fixed density $k_{\mathrm{F}}$. The set of six parameters $\{\alpha, \beta, \gamma\}_{\mathrm{s}, \mathrm{o}}$ was then determined by a least square fit to $U$, i.e. by minimizing

$$
\chi^{2}=\int_{0}^{k_{\mathrm{F}}} d k k^{2}\left[U\left(k, k_{\mathrm{F}}\right)-\left(\frac{\tilde{M}^{*}}{\tilde{E}^{*}} \tilde{\Sigma}_{\mathrm{s}}-\tilde{\Sigma}_{\mathrm{o}}\right)\right]^{2} .
$$

However, such a procedure suffers from a large amount of arbitrariness since one tries to extract two independent functions out of one function. Consequently, the result is strongly influenced by the choice of the trial functions. It is clear that the class of trial functions given by Eq. (42) will generally not provide solutions of Eq. (39), although the insertion of the - a priori unknown - 'correct' amplitude $f_{R}$ should yield the correct results for the self-energies.

To demonstrate this aspect we compare in Fig. 5 the fit procedure according Eqs. (42,43) to the projection method using thereby both, the 'PV choice' and the 'full PV' decomposition. The fitted self-energies are determined from the single particle potential obtained with the 'PV choice'. It is seen that the fitted self-energies show a moderate momentum dependence which is in a qualitative agreement with the 'full PV' representation, but not with the 'PV choice' to which the fit was performed. However, the asymptotic high momentum behavior of the fitted self-energies is completely different from the projected self-energies, independently which choice is used. Also the slope of the curves at low momenta seem to be distorted by the choice of the trial function. The results of Ref. [10] show a similar behavior (which is due to the choice of the same functional $k$-dependence (42)), however, the momentum dependence is a little more pronounced than our fitted results. For a fair comparison one has to be aware that in Ref. [10] and the present work different approximations to the 
Thompson propagator were made. Actually, in the present work the Thompson equation is solved in the two-particle center-of-mass frame, whereas in Refs. [5, 10] it is solved in the nuclear matter rest frame thus avoiding the projection techniques. The main difference is, however, that in Ref. [10] the effective mass entering into the Thompson equation and the effective spinor basis is allowed to be momentum dependent. This states an involved problem which affords a couple of additional approximations. If the momentum dependence of the self-energy is very pronounced one has to go beyond the present approximation scheme with $\tilde{M}^{*}\left(k_{\mathrm{F}}\right)$ in order to include this momentum dependence self-consistently. However, performing the full PV decomposition (which we regard as the most reliable one) the momentum dependence is actually very weak (see Fig. 4). Thus the usage of a constant effective mass $\tilde{M}^{*}\left(|\mathbf{k}|=k_{\mathrm{F}}, k_{\mathrm{F}}\right)$ is well justified. In contrast to [10] where it was argued that the improved self-consistency suppresses the momentum dependence we find that momentum dependence is mainly governed by the representation of the T-matrix or - in the case of Ref. [10] - by the choice of the trial functions.

To illustrate this effect the single particle potential is considered in Fig. 6. It is seen that the different decompositions, 'PV choice' versus 'full PV' representation, yield significantly different results for this quantity. The deviations are due to the different modifications of the effective interaction in the self-consistency scheme which yield also different effective masses. The 'full PV' treatment lowers the potential by about $8 \mathrm{MeV}$ compared to the standard treatment and thus leads to more binding in the equation-of-state. We also included the result of Ref. [10] into this figure. Remarkably, this calculation yields the same result as the present 'PV choice' although the underlying scalar and vector self-energies are completely different. This somehow fortuitous agreement can be understood by the fact that the selfenergies coincide around the Fermi momentum. In addition we show in Fig. 6 the single particle potential obtained as a result of the fit procedure, Eq. (43). $U$ is reasonably well reproduced although the fitted and projected self-energies in Fig. 5 strongly differ. Hence, it is not possible to extract the self-energy components from the single-particle potential in a reliable way. This finding is also supported by a recent analysis where it was shown that the 
fit method breaks down when applied to isospin asymmetric nuclear matter [26]. Recently Müther, Ulrych and Toki [27] also determined momentum dependent scalar and vector selfenergies components directly from the single particle potential (17). There $\tilde{M}^{*}$ was treated as an independent quantity which allows to generate more than one equation for $U\left(k, k_{\mathrm{F}}\right)$

to determine $\tilde{\Sigma}_{\mathrm{s}}$ and $\tilde{\Sigma}_{\mathrm{o}}$. This approach, however, neglects that only the self-consistent $\tilde{M}^{*}$ has a physical meaning. With the method of [27] one also obtains a very weak momentum dependence of $\tilde{\Sigma}_{\mathrm{s}}$ and $\tilde{\Sigma}_{\mathrm{o}}$ similar to the 'full PV' approach of the present work.

Another experimentally accessible observable is the Schroedinger equivalent optical potential [2]. Here the explicit momentum dependence of the self-energies provides an important correction to the in first order linear energy dependence of the optical potential. Adopting the 'PV choice' we already found in Ref. [9] a good agreement with the empirical values of Ref. [25] for the real part up to energies around $800 \mathrm{MeV}$ and an excellent agreement up to the pion threshold for the imaginary part. As can be seen from Fig. 7 where the real part of the optical potential is shown the agreement with the data is even improved at low energies when the 'full PV' representation is used. This also indicates that the depth of the corresponding single particle potential is quite reasonable. At higher energies the two methods yield almost identical results. On the other hand, with the fields as obtained by the fit method and also predicted similar in [10] it is not possible to reproduce the high energy behavior of the optical potential. This will of course have implication when such fields, Eq. (42), are applied to the transport description of heavy ion collisions.

\section{SUMMARY AND CONCLUSIONS}

In the present work we investigated the momentum dependence of the nuclear self-energy in the relativistic Brueckner approach. We applied the standard treatment which projects onto positive energy states and determines the self-energy components by a decomposition of the T-matrix into its Lorentz invariants. The T-matrix is represented by a set of five linearly independent covariants. Since the set of covariants is not uniquely determined in the 
subspace of positive energies one has some freedom in the choice of the representation. It is therefore not possible to determine the scalar and vector parts of the relativistic nuclear selfenergy in a unique way. This ambiguity originates from the fact that the on-shell scattering of positive energy states yields identical values for the pseudo-scalar and the pseudo-vector representation of the corresponding matrix elements and that they connect differently to the negative energy states, see also Refs. [8,19]. In the standard treatment of relativistic Brueckner theory one accounts for this fact by choosing a particular type of a pseudovector representation. To perform this 'PV choice', see Eq. (31), one has to decompose already anti-symmetrized matrix elements into direct and exchange terms which is not free from ambiguities. Applying the Bonn potentials as the bare NN interaction we find that the conventional 'PV choice' leads to a pronounced momentum dependence of the nuclear self-energy. The spatial $\Sigma_{\mathrm{v}}$-contribution of the self-energy is thereby found to be relatively large, in particular inside the Fermi sea, and counterbalances this momentum dependence to some extent on the mean field level. However, this strong momentum dependence makes the standard Brueckner approach questionable since the Thompson equation (or alternative reductions) are iterated using a self-consistent effective mass depending only on the Fermi momentum.

The momentum dependence is found to be completely dominated by the pion exchange. It originates from the pseudo-scalar nature of the pion which is still remnant adopting the 'PV choice' (31) in the conventional manner. To eliminate this insufficiency we represented the T-matrix by a pure pseudo-vector decomposition and called this 'full PV' representation. The 'full PV' representation accounts correctly for the desired pseudo-vector nature of the pion exchange and suppresses the momentum dependence of the self-energy almost completely. The two limiting cases, namely the full pseudo-scalar and the full pseudo-vector representation, set the range of uncertainty in the determination of the self-energy. However, the 'full PV' representation is more consistent with the usual approximation scheme of the Brueckner approach which assumes that the screening of the effective interaction in the medium introduces an additional density dependence, but is only weakly depending on the 
momentum of the particles. We further investigated a frequently used method, namely to determine the scalar and vector self-energy components directly by a fit to the single particle potential. Although this method works reasonably well as long as one restricts to density dependent observables it leads to highly ambiguous results when applied to extract the full momentum dependence of the fields. Thus we conclude that the projection onto covariant amplitudes using thereby a complete pseudo-vector representation is up to now the most reliable way to determine the scalar and vector nucleon self-energy components as long as one works exclusively with positive energy states.

\section{ACKNOWLEDGMENTS}

We would like to thank H. Müther, E. Schiller and H. Lenske for enlighting discussions.

We further thank E. Schiller and H. Müther for providing us with a relativistic Hartree-Fock program which was very useful in order to check the present results.

[1] C.J. Horowitz, B.D. Serot, Nucl. Phys. A464, 613 (1987).

[2] B. ter Haar, R. Malfliet, Phys. Rep. 149, 207 (1987).

[3] P. Poschenrieder, M.K. Weigel, Phys. Rev. C 38, 471 (1988).

[4] C. Nuppenau, Y.J. Lee, A.D. MacKellar, Nucl. Phys. A504, 839 (1989).

[5] R. Brockmann, R. Machleidt, Phys. Rev. C 42, 1965 (1990).

[6] A. Amorin, J.A. Tjon, Phys. Rev. Lett. 68, 772 (1992).

[7] H.F. Boersma, R. Malfliet, Phys. Rev. C 49, 233 (1994).

[8] H. Huber, F. Weber, and M.K. Weigel, Nucl. Phys. A596, 684 (1995).

[9] L. Sehn, C. Fuchs, Amand Faessler, Phys. Rev. C 56, 216 (1997). 
[10] C.-H. Lee, T.S. Kuo, G.Q. Li, and G.E. Brown, Phys. Lett. B 412 (1997) 235.

[11] B.D. Serot, J.D. Walecka, Advances in Nuclear Physics, 16, 1, eds. J.W. Negele, E. Vogt, (Plenum, N.Y., 1986)

[12] P. Ring, Prog. Part. Nucl. Phys. 37, 137 (1996).

[13] F. de Jong, H. Lenske, Phys. Rev. C 54, 1488 (1996).

[14] R. Rapp, R. Machleidt, J.W. Durso, and G.E. Brown, nucl-th/9706006.

[15] H.F. Boersma, R. Malfliet, Phys. Rev. C 49, 1495 (1994).

[16] H. Lenske, C. Fuchs, Phys. Lett. B 345, 355 (1995);

C. Fuchs, H. Lenske, H.H. Wolter, Phys. Rev. C 52, 3043 (1995).

[17] H. Shen, Y. Sugahara, and H. Toki, Phys. Rev. C 55, 1211 (1997).

[18] C. Fuchs, T. Gaitanos, H. H. Wolter, Phys. Lett. B 381, 23 (1996).

[19] F. de Jong and H. Lenske, preprint nucl-th/9709012.

[20] R. Machleidt, Advances in Nuclear Physics, 19, 189, eds. J.W. Negele, E. Vogt, (Plenum, N.Y., 1989).

[21] J.A. Tjon, S.J. Wallace, Phys. Rev. C 32 (1985) 267.

[22] L. Sehn, H.H. Wolter, Nucl. Phys. A601, 473 (1996);

C. Fuchs, L. Sehn, H.H. Wolter, Nucl. Phys. A601, 505 (1996).

[23] M.L. Goldberger, M.T. Grisaru, S.W. Mac Dowell, and D. Wong, Phys. Rev. 120 (1960) 2250.

[24] R. Fritz and H. Müther, Phys. Rev. C 49 (1994) 633.

[25] S. Hama, B.C. Clark, E.D. Cooper, H.S. Sherif, R.L. Mercer, Phys. Rev. C 41 (1990) 2737.

[26] S. Ulrych, H. Müther, Phys. Rev. C 56 (1997) 1788.

[27] H. Müther, S. Ulrych, H. Toki, nucl-th/9711010. 
TABLE I. Binding energy per particle $E$, Fermi momentum $k_{\mathrm{F}}$, reduced effective mass $\tilde{M}^{*}$, and compression modulus $K$ for nuclear matter at saturation density employing the various representations of the T-matrix (41). As the bare NN interaction the Bonn A potential was used.

\begin{tabular}{ccccc}
\hline \hline & $\begin{array}{c}k_{\mathrm{F}} \\
{\left[\mathrm{fm}^{-1}\right]}\end{array}$ & $\begin{array}{c}\mathrm{E} \\
{[\mathrm{MeV}]}\end{array}$ & $\begin{array}{c}\tilde{M}^{*} \\
{[\mathrm{MeV}]}\end{array}$ & $\mathrm{K}$ \\
\hline PS & 1.45 & -17.70 & 455.6 & 335 \\
PV choice & 1.41 & -15.81 & 538.3 & 275 \\
full PV & 1.42 & -16.59 & 648.8 & 245 \\
Ref. [5] & 1.40 & -15.59 & 564.3 & 290 \\
\hline \hline
\end{tabular}




\section{FIGURE CAPTIONS}

Fig. 1: Pion contributions only to the nucleon self-energy in the Hartree-Fock approximation. The self-energies determined in the pure pseudo-scalar (PS) or the pure pseudovector (PV, multiplied by a factor 10) representation are compared to results obtained within the 'PV choice'. In all calculations the nuclear matter density is chosen as $\rho=0.166 \mathrm{fm}^{-3}$ and the pion coupling constant and form factor from the Bonn A are used. Solid lines represent the scalar, dashed lines the vector self-energy.

Fig. 2: Momentum dependence of the nucleon self-energy in the relativistic BruecknerHartree-Fock approach using the 'PV choice' (see (41). The nuclear matter density is chosen as $\rho=0.166 \mathrm{fm}^{-3}$ and the Bonn A potential is used. Solid lines represent the scalar, zero-vector and space-like vector components $\Sigma_{\mathrm{s}}, \Sigma_{\mathrm{o}}$ and $k_{\mathrm{F}} \Sigma_{\mathrm{v}}$; dashed lines represent the corresponding reduced fields into which the $\Sigma_{\mathrm{v}}$-term is effectively included (16).

Fig. 3: Influence of the various meson exchange contributions on the nucleon self-energy (scalar part). The solid line corresponds to the full calculation (Bonn A), the dotted line to $\sigma \omega$ and the dashed line to $\sigma \omega \pi$-exchange only. In all calculations the 'PV choice' is used.

Fig. 4: Comparison of the reduced self-energy components, Eq. (16), obtained by the various decompositions of the T-matrix (see (41). Solid lines correspond to the 'full PV' representation, dashed lines to the 'PV choice' and long-dashed lines to a pure PS representation. The shaded area indicates the range of uncertainty spanned by the 'full PV' and the PS representation, Eq. (21). The nuclear matter density is chosen as $\rho=0.166 \mathrm{fm}^{-3}$ and the Bonn A potential is used.

Fig. 5: The reduced self-energy components obtained with the projection method using the

'PV choice' (long-dashed, see (41)) are compared to the respective fields obtained by 
a fit to single particle potential (42) (dotted). In addition the results with the 'full PV' representation (solid) and those of Ref. [10] (dot-dashed) are shown. The nuclear matter density is chosen as $\rho=0.166 \mathrm{fm}^{-3}$ and the Bonn A potential is used.

Fig. 6: Single particle potential in the relativistic Brueckner-Hartree-Fock approach with the Bonn A NN interaction at $\rho=0.166 \mathrm{fm}^{-3}$. The results of the projection method with the 'full PV' representation (solid line, see (41)) and using the 'PV choice' (dashed line) are shown. The dotted line represents the single particle potential obtained by the fit procedure (42) and (43) to the result of the 'PV choice'. For comparison also the calculation of Ref. [10] is shown.

Fig. 7: Schroedinger equivalent optical potential for Bonn A at $\rho=0.166 \mathrm{fm}^{-3}$. The results of the projection method with the 'full PV' representation (solid line, see (41)) and using the 'PV choice' (dashed line) are shown. The dotted line represents the result obtained with the fit procedure (42) and (43) to $U$. The empirical values (diamonds) are taken from [25]. 


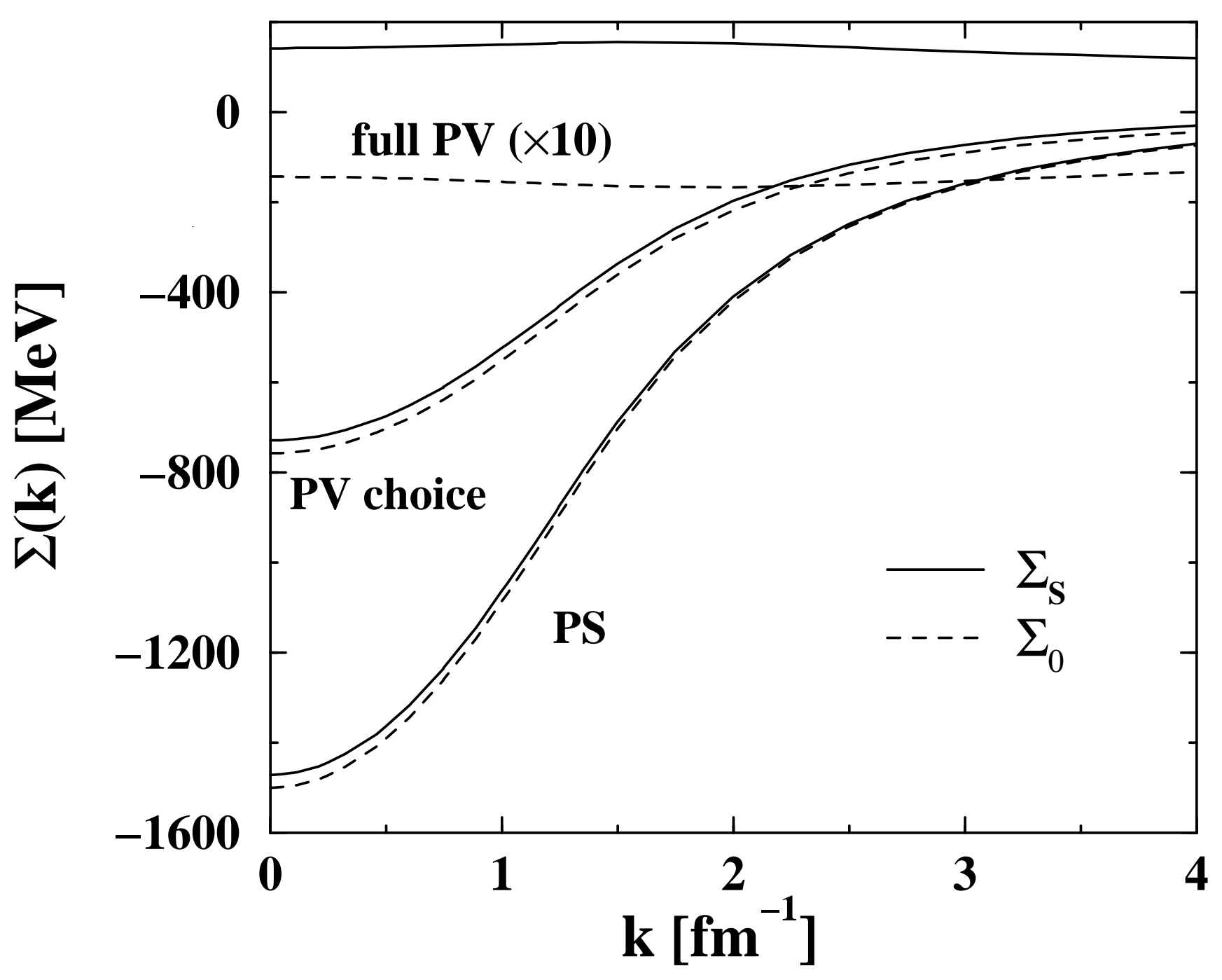

FIG. 1. 


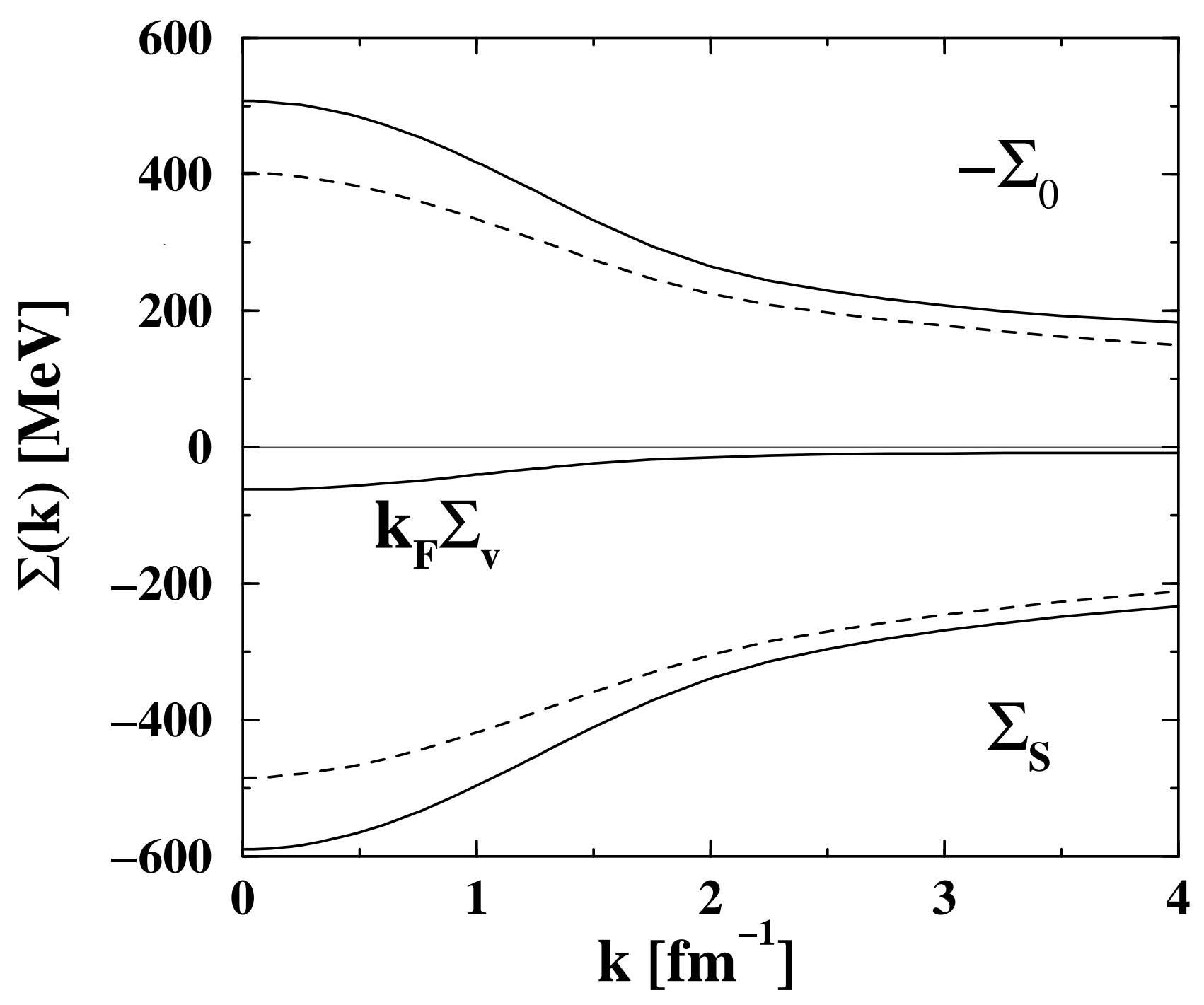

FIG. 2. 


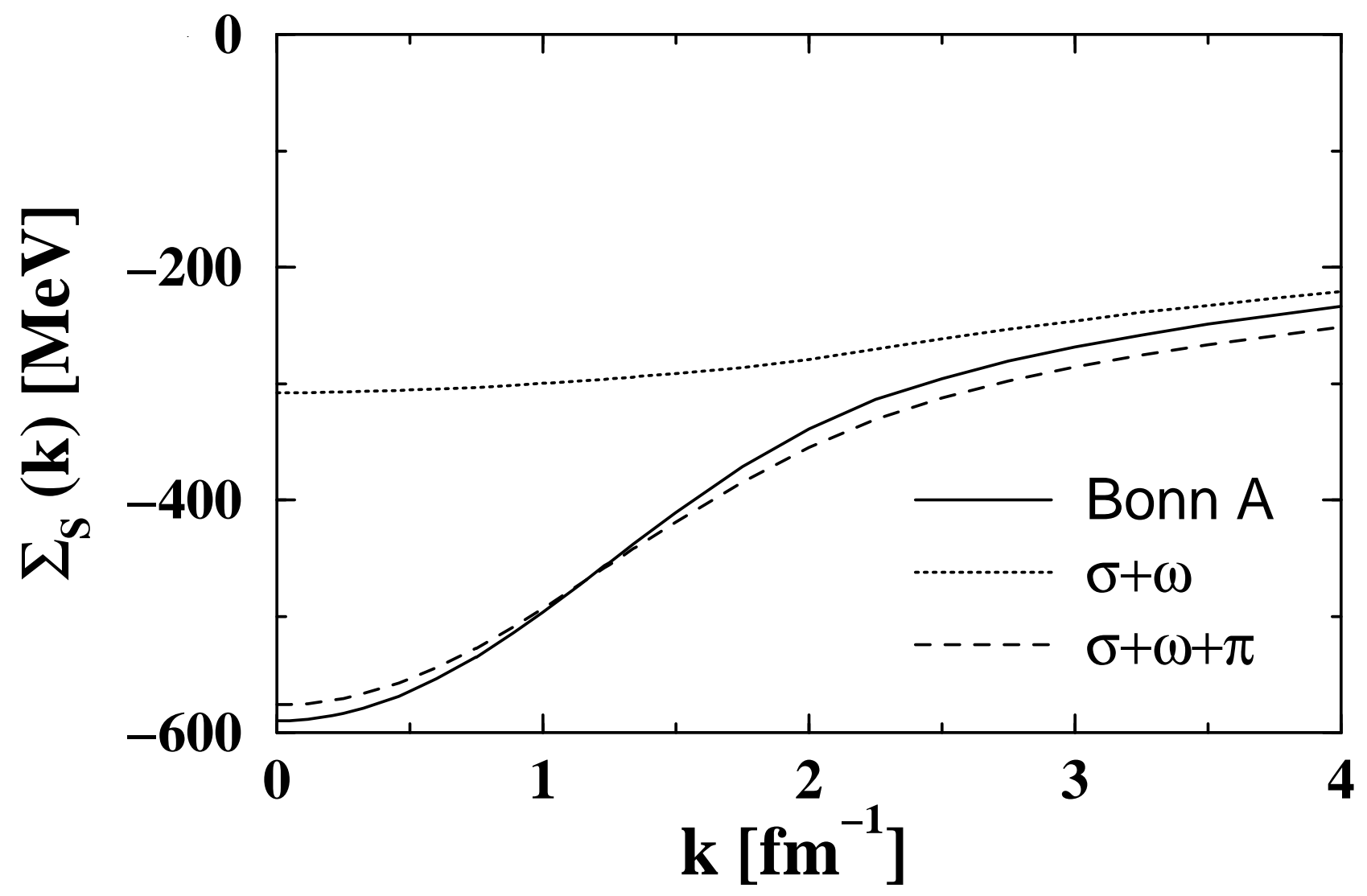

FIG. 3. 


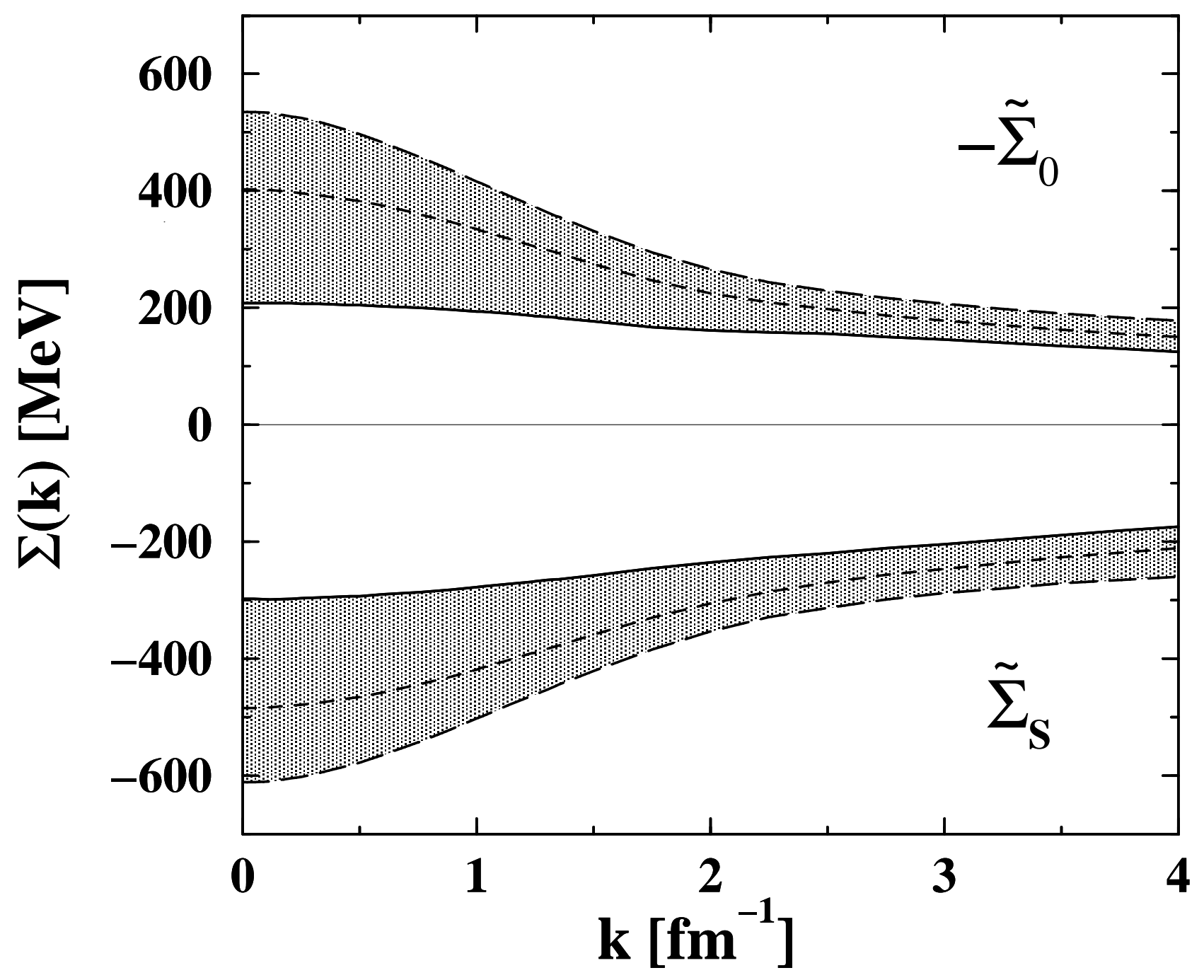

FIG. 4. 


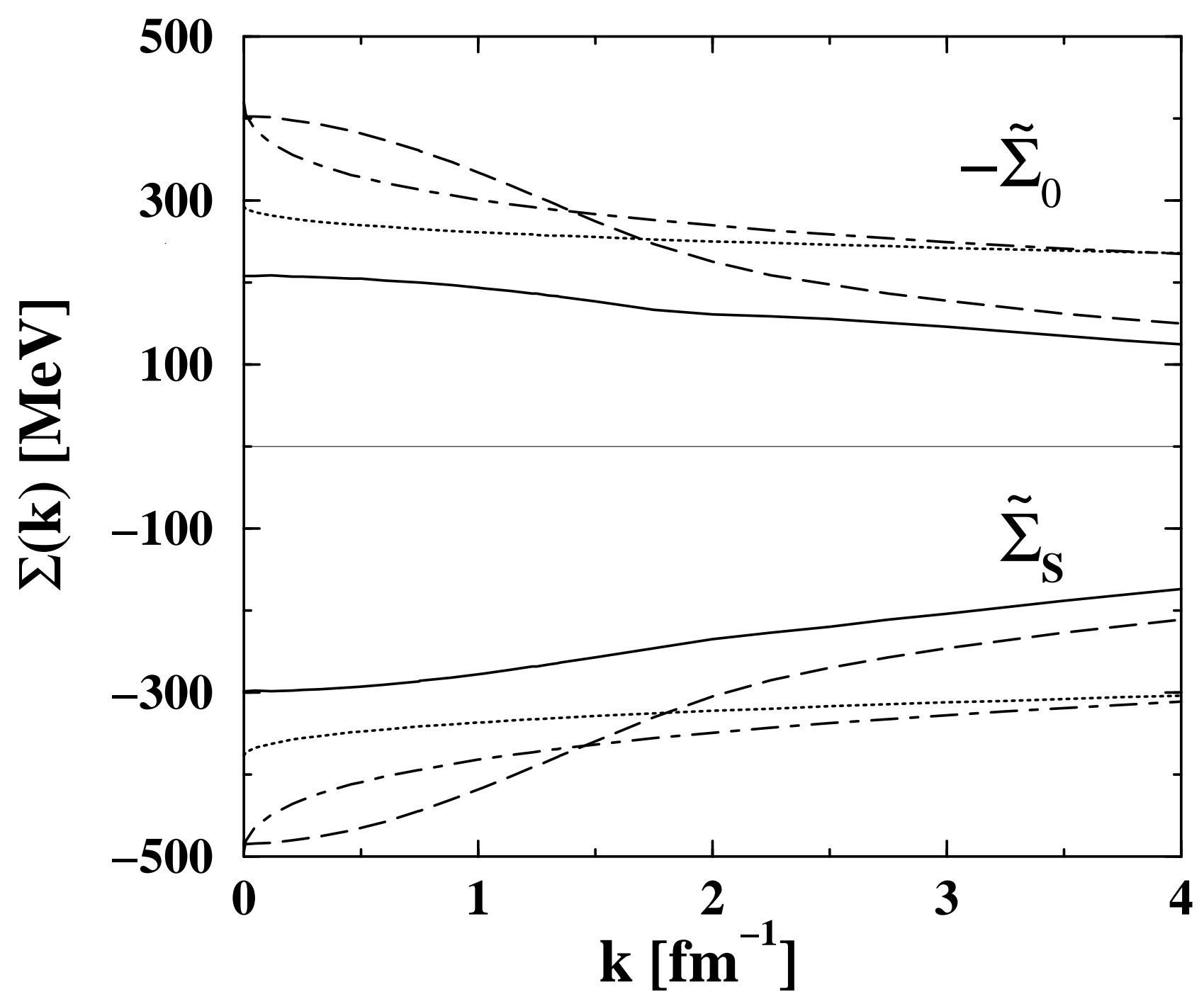

FIG. 5. 


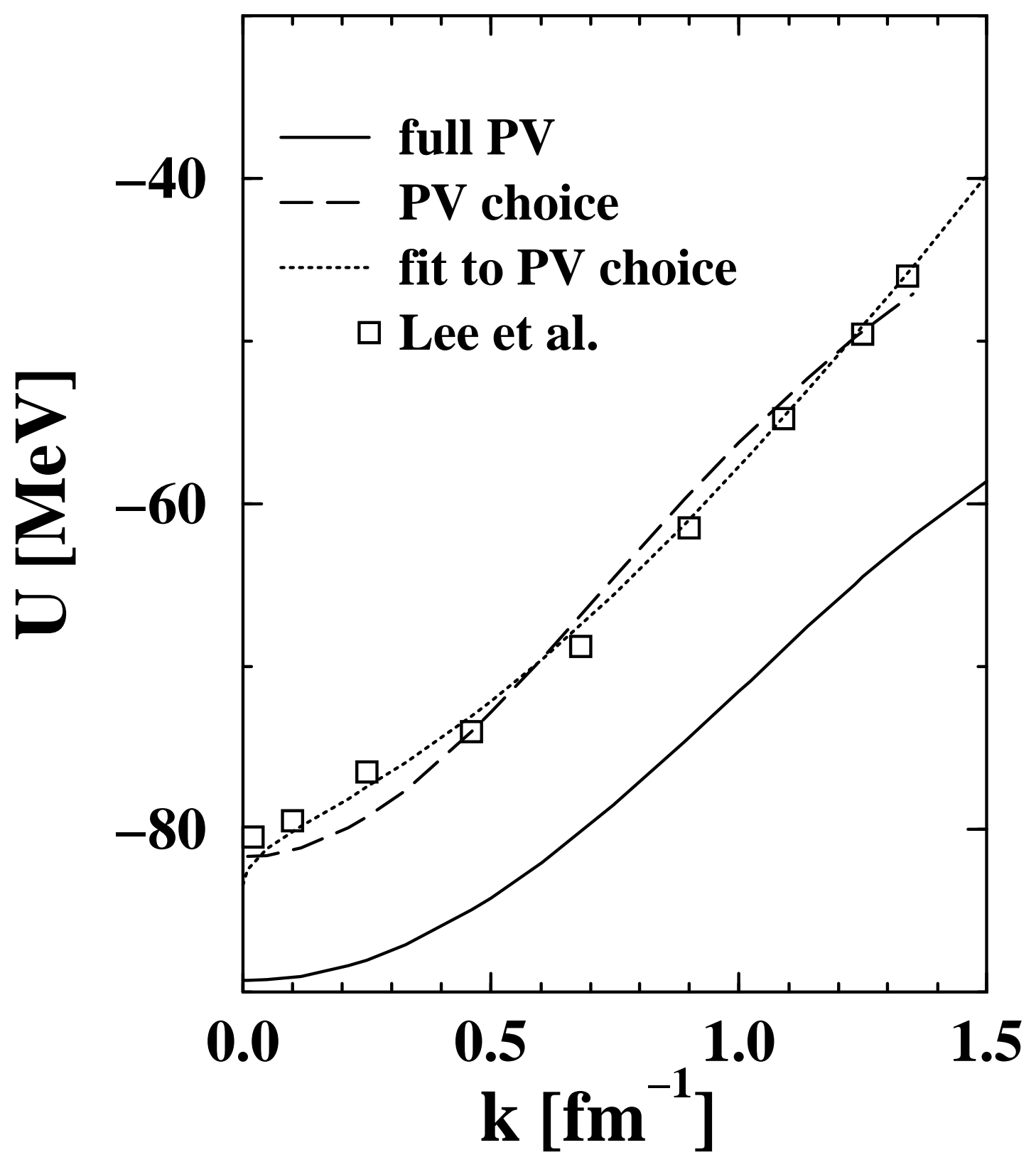

FIG. 6. 


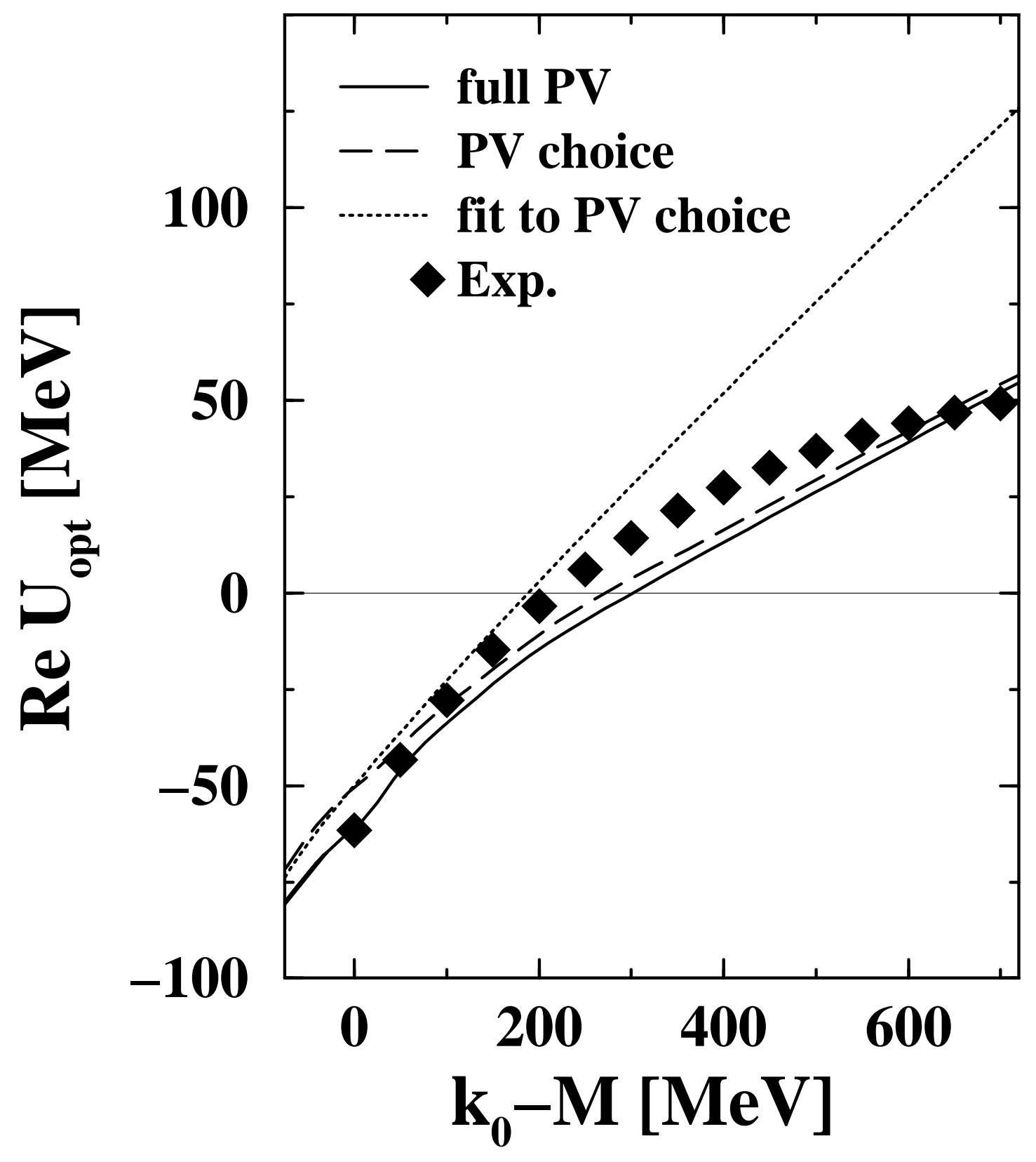

FIG. 7. 